\title{
HUMANS 2.0
}

\section{Writing the future of human evolution}

\section{GEMMA MARFANY}

Can humans control the future evolution of our species? Based on current knowledge in genetics, one can infer and extrapolate what may happen in the near future. After all, if we are to predict the future, we must first understand the foundations of our present. To answer the first question, I will briefly present what we know about our genome and whether we have enough data to infer who we are (known as the genotype-phenotype correlation), then I will present new technological advances and their potential impact on our evolution.

Keywords: genome sequencing, genetic editing, human genetic modification, genetic determinism, bioethics.

\section{DO WE KNOW WHY WE ARE THE WAY WE ARE?}

\section{Our genome}

When one studies the genetic and molecular bases of the human phenotype (for example the causes of rare diseases) one enters into the field of both Mendelian heritage and of the genetics of diseases and other more complex traits, such as behaviour. Thanks to the increasing affordability of massive sequencing, we can now easily sequence our genome. When sequencing a genome, the number of genetic variants identified from what is considered the reference genome is very high, around four or five million (The 1000 Genomes Project Consortium, 2015). However, the objective is usually to identify the genetic cause of a disease in one given patient, so instead of sequencing the genome, we choose to sequence the exome, that is, we focus on protein or RNA-coding regions, from which we can more easily infer the potential biological effect of the identified variants. Even if we simplify the analysis, the interpretation of the exome is not direct, because on average between 20,000 and 30,000 variants are identified with respect to the reference human exome. These variants largely explain our diversity, but trying to interpret everything is dizzying (because we still know very little), so we focus on specific diseases or specific regions of the genome.

\section{Mendelian traits and diseases}

Since humans became human, they have been fascinated by the fact that descendants resemble their parents. Also, since ancient times humans have known how to cross-breed plants and animals, but the laws behind the results of these crossings were impenetrable until a nineteenth-century monk, Gregor Mendel, analysed the data statistically to try to understand how certain traits were transmitted in peas. Mendel's laws are intellectually attractive and satisfying, but few of the traits seen in organisms

follow purely Mendelian inheritance patterns. We know that there are genetic phenomena and environmental factors (see below) that cannot always be directly inferred, and this shows that the genotype-phenotype correlation - using a gene sequence (genotype) to directly infer the trait it determines (phenotype) - is neither easy nor simple.

Many of our traits are explained by the effect of more than one gene; that is, we need more than one genetic instruction to perform certain cell functions, and this means that mutations in many different genes can ultimately cause the same phenotype. For example, hereditary blindness in humans is related to over 300 genes. ${ }^{1}$ Focusing only on those that cause

\footnotetext{
${ }^{1}$ http://www.dbgen.org; https://sph.uth.edu/retnet/
} 
blindness by interfering with ciliary function (the cellular organelle that acts as an external sensor) highlights around fifty causal genes. However, the mutations in these ciliary genes, when serious enough, not only cause blindness but also affect many other organs and functions, such as the cochlea, kidneys, development and internal placement of organs, and neural tube closure, among others. So, one gene can perform many functions and the network of interactions with other genes is by no means simple.

\section{Genetic factors and interaction with the environment}

For many traits, genes contribute quantitatively. In other words, each genetic variant adds or removes something from the picture and, in combination with environmental interactions, determines the final result. For traits such as height or body weight, it is evident that genetic and environmental factors play a role. One only needs to think about the Maasai, who are always tall despite their nutrition status, even though a very well-fed young person who exercises can become taller than they would without access to a healthy diet. Thus, the genotype determines the range of responses and the possible spectrum of phenotypes, and the interaction with the environment merely determines the result within this range.

We often talk about susceptibility variants or genetic predisposition to explain the increased risk that some people have of suffering certain diseases. The same disease may have a very strong Mendelian component, which explains a small number of cases; but it may also be associated with many variants that are common in the population which, along with certain environmental stimuli, increase the risk of suffering a disease, as it happens with hypertension, osteoporosis, hypercholesterolemia, Parkinson's disease, or Alzheimer's disease. Thus, when genetic and environmental factors are combined, we do not always know how to disentangle their exact relationships.

In cases of hereditary cancer, we can inherit germline mutations in certain genes such as $B R C A I$ and $B R C A 2$ that greatly increase the likelihood of developing breast cancer. However, these genes do not absolutely determine this risk because additional random mutations in the cells of the body are needed for the disease to develop. Therefore, in advance, we can only warn about the risk of suffering from a given type of cancer, but we cannot categorically predict whether the carrier will develop it or not. The environment is also important, as it can accelerate the mutational process: consider, for example, lung cancer and its relationship with tobacco smoke, which contains several carcinogenic components.

\section{Unexpected genetic changes}

Thanks to the sequencing of many human genomes, we now know that much of our diversity lies in the dose of genetic material we inherit. Many chromosome regions comprising one or a few genes can become duplicated within the same chromosome, so some people have more or fewer copies of certain genes. Consequently, the proteins they encode may be more or less abundant.

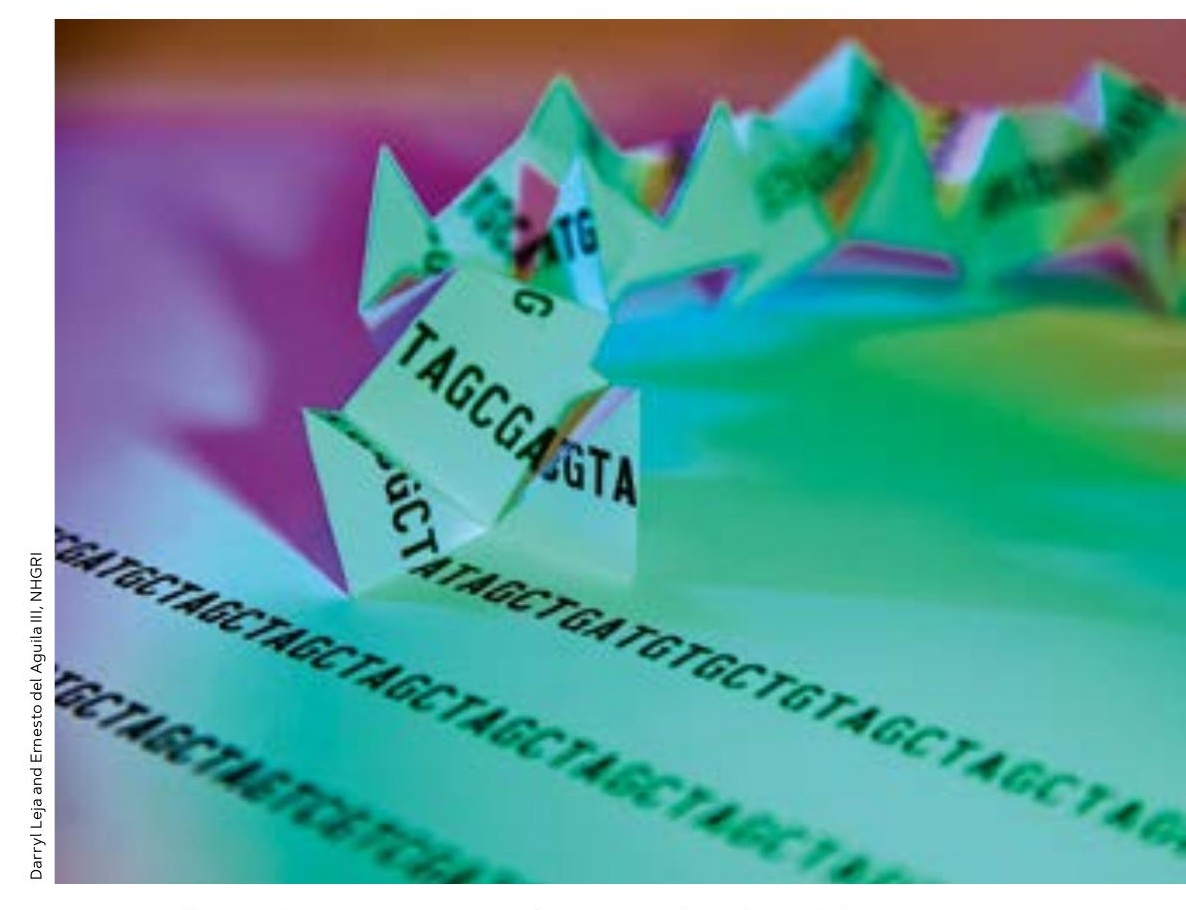

Sequencing genomes has now become much easier and cheaper. Because the number of genetic variants identified is very high (between four and five million), and the objective is usually to identify the genetic cause of a disease in a given patient, instead of sequencing the genome, we choose to sequence the exome. That is, we focus on protein or RNA-coding regions from which we can more easily infer the potential biological effect of the identified variants. 

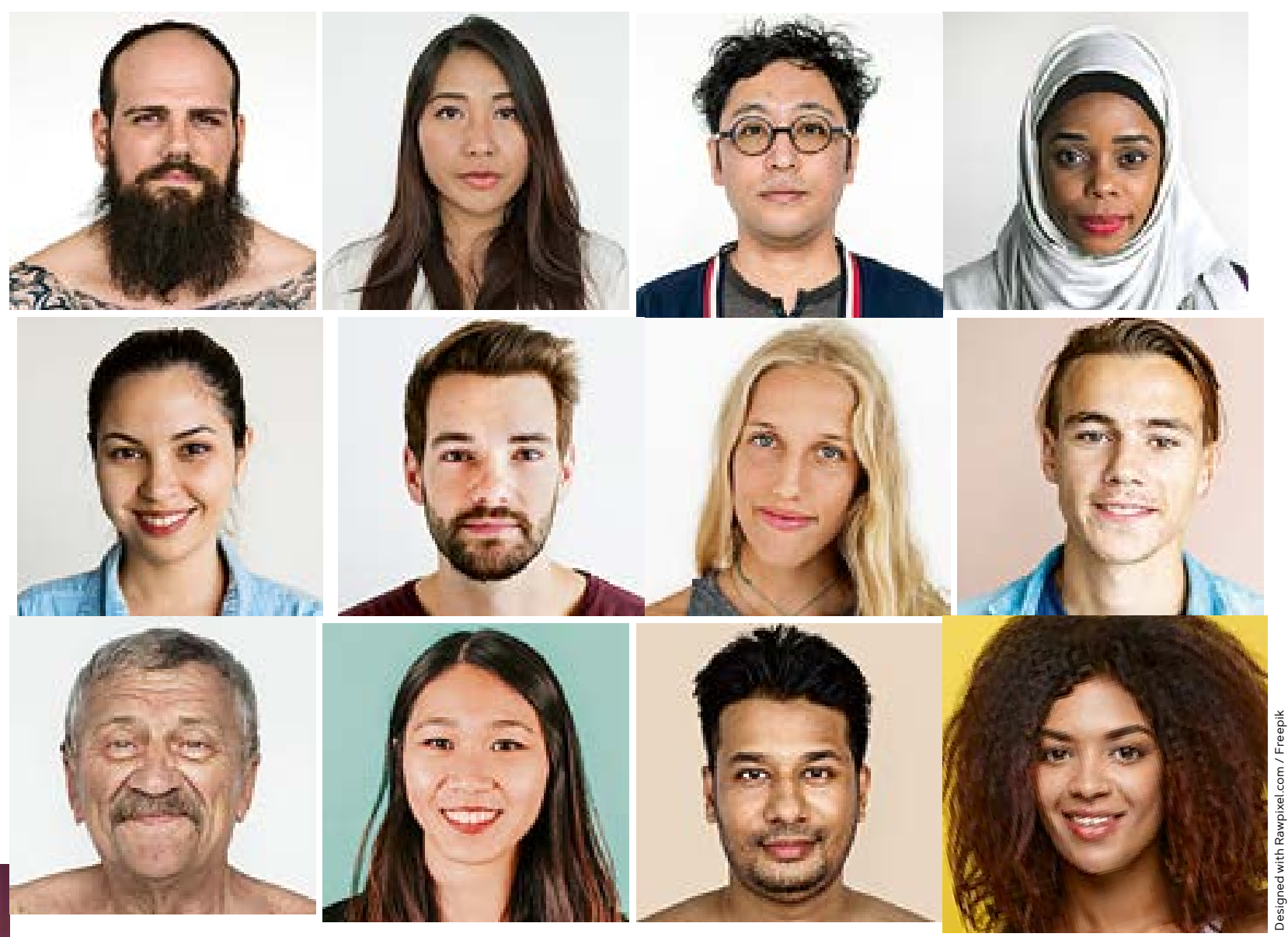

On average, between 20,000 and 30,000 variants are identified with respect to the reference human exome; these variants largely explain our diversity.

Indeed, copy number variants (CNV) are believed to be one of the most important genetic reasons for the diversity of some cognitive and behavioural traits. For example, CNVs have been associated with cognitive impairment, autism spectrum disorders, genetic susceptibility to mental disorders, and responses to psychotropic drugs or medications which act on the central nervous system.

These are not the only surprises in human genetics, because many diseases are also related to intellectual capacities not dependent on classical Mendelian inheritance. Massive sequencing is helping to diagnose cases of rare or ultrarare diseases when there is no family precedent; the assumption was always that their genetic origin is a recessive condition resulting from the inheritance of genetic mutations from both parents. But now that TRIOS analysis (two parents and the son or daughter) is possible, we are realising that there are many dominant ailments and that mutations occur de novo: neither parent carries the mutation presented by the progeny (Ku et al., 2013). These de novo mutations can occur during the meiotic formation of the parents' gametes (in a spermatozoon or ovum) so all of the offspring's (the patient's) resulting cells contain the mutation; they can also occur somatically in the embryo, so only a few of the patient's cells have the mutation, making the patient a mosaic of mutated and unmutated cells. Indeed, these de novo mosaic somatic mutations have been found in patients with autism spectrum disorders (Lim et al., 2017). The seriousness of the mutation and the percentage of cells affected will determine the severity of the disease, so it is difficult to define the exact phenotype of a mosaic individual in advance.

\section{Sequencing our individual genomes}

Sequencing our genomes (or exomes) gives us a measure of our genetic diversity, but apart from identifying our genotype variants, it is also important to know what the phenotype is. What do we know about our genome and what can we infer? By analysing mitochondrial DNA and the Y chromosome, we can discover the ethnogeographic background of the person. 
We can also predict quantitative traits, such as skin, hair, and eye colour or the general shape of the face. We have even discovered that the Homo genus is not monophyletic but rather, modern humans are the product of crossbreeding with other hominins such as Neanderthals and Denisovans. Indeed, chromosomal fragments can be found in our genome that come directly from these already extinct Homo species which still live inside us (Vernot \& Pääbo, 2018).

Genetic diagnosis using massive sequencing also allows us to identify many of the mutations that cause Mendelian diseases, but we can only offer genetic predisposition values for most diseases that affect us. In other words, probabilistic lists of traits: a person might have a $30 \%$ probability of suffering from prostate cancer, $50 \%$ for colon cancer, $10 \%$ for breast cancer, $10 \%$ for cardiovascular problems, and have a $60 \%$ risk of suffering from myopia, etc. Yet, we still do not know what to do with these data in the absence of knowledge of how to properly use them; nor do we know who has access to them and how they will be used. Indeed, perhaps we should prepare ourselves for the coming avalanche of genetic information: I think it is very likely that we, and future doctors, will use this information to prevent or delay certain diseases. Some medical colleges already claim that doctors should have access to all the patient's incidental results (results related to pathologies other than the ones being diagnosed) when data to diagnose patients affected by a genetic disease is generated by massive sequencing; they argue that any findings, especially cancer-related mutations or those that could directly impact health, should be communicated to the patient (Kalia et al., 2017).

\section{The genetic horoscope}

Understanding what we are like and how we will become, the life we should lead and the partner we should choose, and what our children will be like, etc., are just some of the promises made by companies that offer direct-to-consumer genetic testing. This supposes that the genetic inferences between genotype and phenotype are known, that everything we are is genetically predetermined, and that knowledge of our genome sequence can be used to directly infer a picture of our future selves (Roukos, 2014). This would be like saying that, with all the pieces of a giant three-dimensional puzzle and an immense book of instructions (that can change over time), we can see the result even before starting to read the book.

We have already mentioned that genetics confers potentials and gives us the range of responses, but the relationship between our genetic variants and more subtle phenotypes is not direct. This is because many of our traits are the product of numerous genetic instructions that interrelate with each other and the environment, and we still do not know how to extract all this information exclusively from our genome. The feeling is that we can still only see the tip of the iceberg.

\section{CHANGING THE FUTURE}

\section{The mirror of our genome}

What can we do with all these genetic data? What does it tell us about our evolution? If we consider that the natural selection of organisms acts on their descendants to transmit the most successful genetic combinations, also affecting the number of descendants produced, then humans have changed the terms of natural selection. As a society, we can take care of individuals with disabilities and functional diversities, who would have barely survived without modern medicine or technology, and so these individuals can
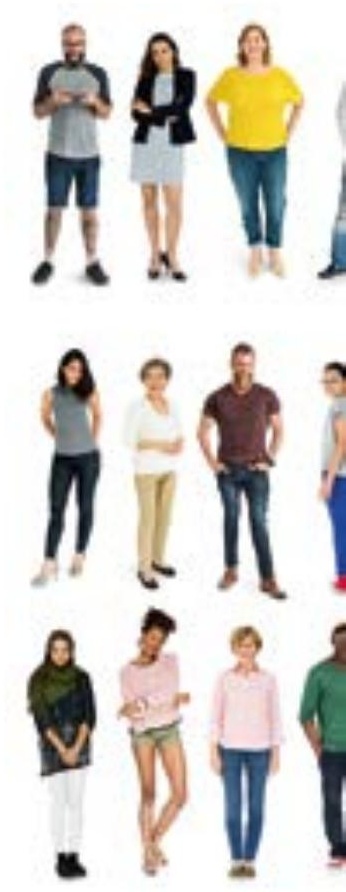
themselves now have offspring; conversely, through war or child sex selection, humans eliminate other individuals who might have survived in past times. Highly capable human beings can decide to devote their entire lives to art, science, or politics, but not to having any children, thus removing their gene combinations from human «genetic heritage».

Furthermore, human living conditions have changed enormously and it is difficult to predict which genes will be selected. The set of genes that we humans have today is the result of our previous history. They come from small human populations with few gene combinations, some of which expanded when the environmental conditions allowed the population to increase. These periods were followed by genetic bottlenecks caused by infections, natural disasters, and migrations when the environmental conditions were very harsh. Indeed, the remnants of this type of selection remain inside us, for example, in the high frequency of the allele that causes sickle cell anaemia in malaria-endemic areas. This allele can be present in more than $25 \%$ of the population, even though the disease is lethal in homozygotes, and heterozygotes ${ }^{2}$ suffer cardiovascular complications. Another example are the mutations that cause haemochromatosis, which allow iron to be recycled more efficiently, resulting in an increase in blood iron concentration, causing the formation of iron deposits in peripheral tissues. This mutation has most probably been selected favourably because it gives an advantage to women, who need greater amounts of iron because of periodic menstrual bleeding and pregnancies, especially since the Neolithic when continuously beyond childhood. Thus, individuals with this mutation can continue to drink milk from other mammals during the years they are growing and after their mother stops breastfeeding them because she has a new baby, thus increasing the carrier's survival rate. Not all humans have inherited the mutation and so some people cannot drink milk when they are older

\footnotetext{
human diets became rich in cereals and poor in iron (McCullough, Heath, \& Smith, 2015).

Mutations in the lactase promoter have also been favourably selected. ${ }^{3}$ These mutations have occurred on at least two independent occasions and allow the lactase gene to maintain its expression because they are unable to digest milk sugar. This already indicates that mutations are not always undesirable or harmful but depend on external conditions and have even been favourably selected because they have improved the survival of heterozygous carriers (Gerbault et al., 2011).

Sometimes mutations may be desirable in young people but not in adults. For example, it is very likely that mutations that facilitate high blood cholesterol levels were favourably selected. Cholesterol is the main agent involved in atherosclerosis and severe cardiovascular problems in adults but is also required to maintain cell membrane lability, is the base component of sexual hormones, and is required for correct foetal neural tube closure (Santander et al., 2013).

(1)

FiFi:

\section{«Gene therapies are extremely precise and only suitable for caused by a specific genetic defect»}


Thus, our current genome is a mirror of our past, but if we look carefully, many of these mutations are not needed in our modern industrialised society anymore: we have eradicated malaria from Europe and the United States, we can take iron supplements if necessary, and babies do not depend exclusively on milk. Therefore, these factors no longer determine our survival, nor the set of genes we will pass on to future humans.

\section{New therapies and implants}

Humans in today's society choose how many children they want to have, if at all. We then try to maximise the survival of the few children we have by applying all the technological and medical advances within our reach, including antibiotics, surgical interventions, prostheses, and organ or marrow transplants. In addition, current advances now allow us to envision the cure or alleviation of hereditary genetic diseases that were, until recently, incurable.

We now hear about precision biomedical therapies such as gene therapy and cell therapy. Gene therapy attempts to correct the effect of a mutation or disease by introducing genetic information. Classically, therapeutic viruses containing the correct gene have been developed and introduced into the cells of patients with incurable diseases. The first commercial therapies are beginning to emerge, for example, to treat blindness in children (Apte, 2018) and there are already several clinical trials underway that indicate that more gene therapies will soon be within our reach, offering hope where previously there was none. However, these therapies are extremely precise and only suitable for patients who have a disease caused by a specific genetic defect. This is a limitation (hence the high price) and may make them accessible only to a few, which would increase the obvious worldwide inequality in access to healthcare.

In cell therapy (for example, bone marrow transplants), healthy cells are introduced into the patient to correct or cure a disease, but there is a shortage of compatible donors. Moreover, if healthy, corrected cells can be generated from the same patient, they can be re-implanted into the right organ to correct the disease without being rejected. The development of induced pluripotent stem cell (iPSC) technologies has allowed the field to explode and is expected to combine both gene and cell therapy techniques. So far, one of the most spectacular cases of this combination was the effective healing of a child with mutations in the laminin 332 gene, suffering from butterfly skin disease. Skin stem cells from the patient were infected with therapeutic viruses containing the correct laminin 332 gene and used to generate «sheets» of corrected skin cells in the laboratory, which were then used for transplantation (Hirsch et al., 2017). The child now has normal skin and can be considered cured, even though he remains homozygous for the mutation and will pass on this gene to his natural offspring.

Likewise, we can think of prostheses and implants - some extremely sophisticated - as entering the sphere of cyborgs. Indeed, some people have implanted sensors under their skin that allow them to communicate with intelligent devices. Sensors can also be external and much less invasive: for example, worn on the skin as temporary tattoos containing integrated circuits which allow us to control devices and give commands such as watering plants or turning

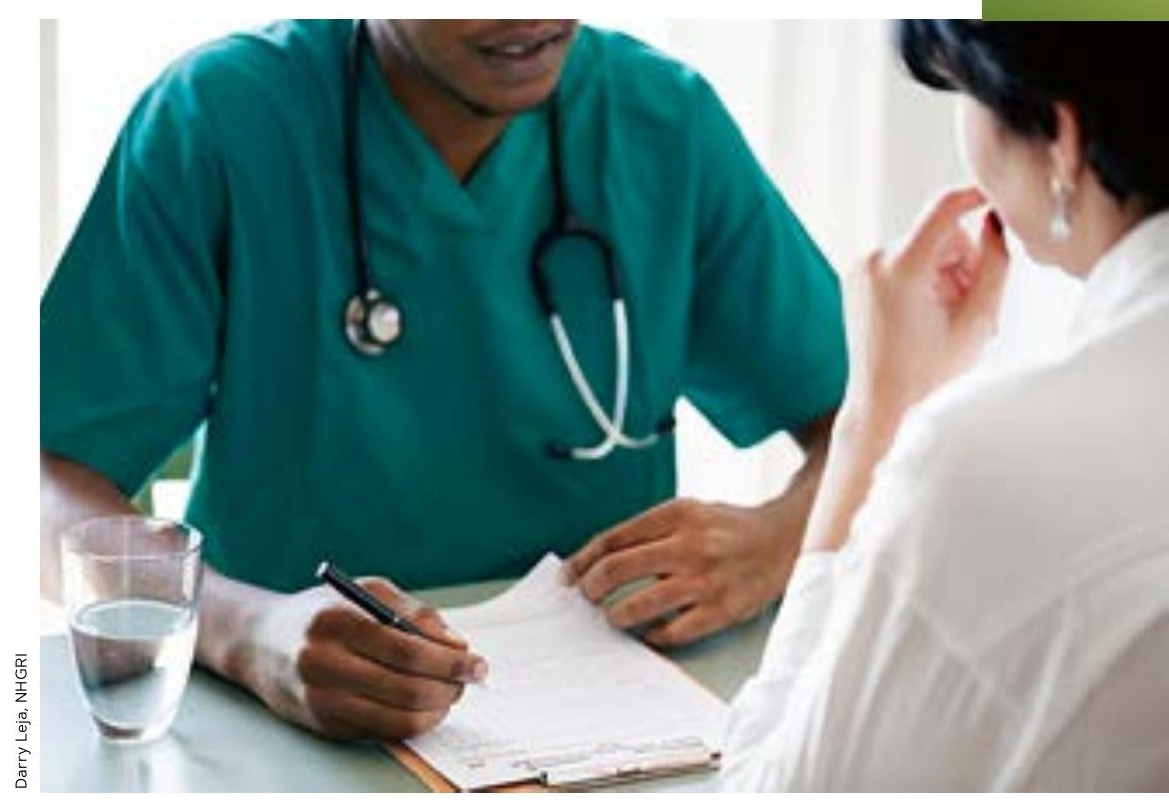

It is very likely that future doctors will use information generated by diagnoses made with massive sequencing techniques to prevent or slow down certain diseases. Some medical colleges already claim that doctors should communicate the results of a genetic diagnosis to the patient, even when this information is not related to the initial pathology, because they can provide insights into mutations associated with the probability of suffering other diseases.

\section{«New genetic editing technologies based on CRISPR/Cas9 and the like are fast, efficient, simple, modular, and, above all, highly targeted»}




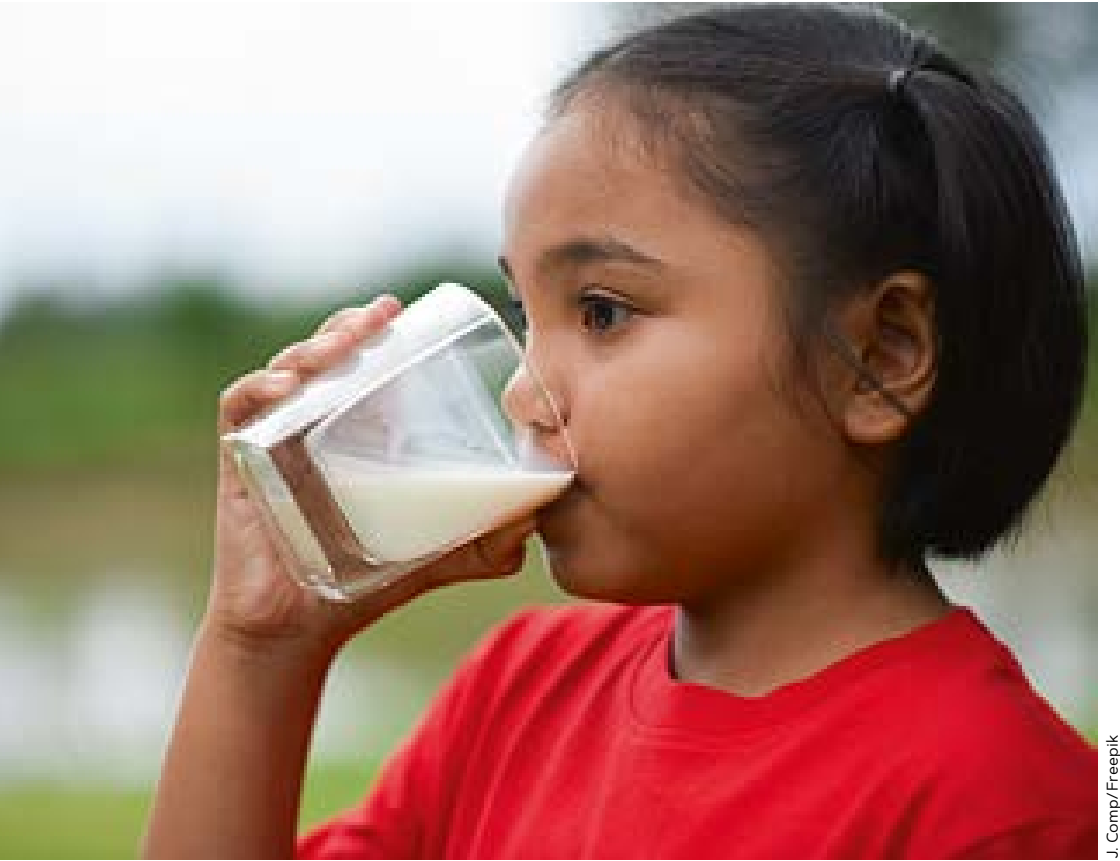

Mutations are not always undesirable or harmful. For example, carriers of a mutation in the lactase promoter can continue to drink milk from other mammals during their growing years and after their mother stops breastfeeding them, which increases the carrier's survival rate.

on the heating, with only the slightest contact (Beans, 2018). There are also health sensors, which measure blood glucose concentration in real time and send signals to deliver homeostatic insulin self-injections, just as our pancreatic beta cells do. All this is now feasible, and these advances only require technological improvements and lower costs so they can be made available to everyone; these implants and improvements could make up for our shortcomings or even add capabilities to our body, but they would not change the genome of future humans.

\section{EDITING THE GENOME: CORRECTION OR IMPROVEMENT?}

I have deliberately left the discussion of what I believe will completely change the future of our genome until last: the ability to precisely modify our genome to encode a specific sequence and introduce new information into it. This possibility has always existed using genetic engineering techniques. In fact, we have already used them to modify other organisms, in some cases at the cost of considerable time and expense (for example, to generate «knockout» or «knock-in» mice), but they have never been effective and affordable enough, nor can we sufficiently control them to risk modifying our own germlines. However, new genetic editing technologies based on CRISPR/Cas9 and the like are fast, efficient, simple, modular, and, above all, targeted precisely.

CRISPR/Cas9 is a bacterial system defence against viruses, which has been harnessed as a biotechnological tool that combines the specificity of nucleic acid sequence pairing with the catalytic efficiency of proteins (Mojica \& Montoliu, 2016). In its most classical application, this editing system allows chromosomal DNA to be broken at a very specific pre-selected location, so that the cell's attempts to repair the damage generates mutations allowing the genetic instructions encoded in a gene to be easily and permanently broken; system modifications would cause the cell to repair DNA conservatively and recombine, thus correcting the mutation and restoring the gene's correct sequence. This system can also be used to introduce genetic variants into DNA that no other human has, or to insert new genes not present in our genome before. Furthermore, by modifying the Cas9 protein and adding new domains to it or changing the ones it already has, we can make a specific gene express itself or, conversely, repress or silence it (Wang, La Russa, \& Li, 2016).

Everything we have mentioned involves DNA editing and changes in the genotype, but what do these changes imply at the phenotype scale? It means that we can correct the genetic mutation that causes a hereditary disease directly in the patient's body like more conventional gene therapies, but we could also do it by modifying sperm or egg cells, thus eliminating certain genetic variants or mutations that we consider undesirable. In the latter case, we would be modifying all the DNA of a new individual and, therefore, the changes would be permanent and could be transmitted to the individual's descendants.

We must reflect upon this further. Do we have as much control over this technique as we think we do? What if the editing system does not operate perfectly and (in a phenomenon known as «offtarget») unexpectedly modifies other parts of the genome? When modifications are made in vitro they 
can be better controlled, but what about in vivo modifications, made directly in an individual? When we talk about generating permanent changes in the genome of future individuals, we must make sure that we do not inadvertently cause a series of problems.

To reflect upon this a bit more, what do we mean when we speak of a serious mutation and a severe illness? We all know that some diseases are lethal and there is no argument that they require therapy, but does myopia magna count as a severe disease? What about genetic susceptibility to breast cancer? Or a genetic predisposition to Alzheimer's disease? Do we also want to change those variants? Should we do so in individuals who have inherited the genes but have not yet developed the disease? Do we want to permanently correct these genes and modify the DNA that future individuals will inherit in order to eradicate these diseases? Or should we wait for people to manifest the disease and then act? What should we do with autoimmune diseases? If we get rid of the genetic variants that increase the risk of suffering them, we may also eradicate variants required to elicit an immune response in other circumstances or against certain parasites. Perhaps we decide to eliminate the genetic variants that predispose us to drug addiction, but it turns out that these variants are also relevant in innovative and creative personalities.

What do we do? What do we

want to do? We think we have the answer, but we do not even know all the genetic interactions involved. We might fix one thing and ruin another. We still do not know, because, as we discussed, the relationship between genotype and phenotype is not always direct, and we do not fully understand it.

This is if we talk about diseases, but we could also apply this technology to perform genetic enhancements. Why not have more muscle mass, be taller, have lighter or darker skin? Again, these genetic modifications could be made in adults, but also in gametes, leading not only to the genetic selection of children, but also to the modification of genes so those who inherit them never become bald, have perfect eyesight, are athletic, have a high intellectual quotient, or have an excellent musical ear. Do we want custom-made babies? Can we even make them? At what cost? If everyone has access to this technology, perhaps we would be paying the extremely high price of dangerously narrowing down our genetic diversity.
But if its cost is too high, perhaps it will only benefit a small number of people. We must carefully consider these questions. Regardless, science fiction predicts that leaving genetic modification technologies within the reach of only a few would lead to the generation of privileged social elites.

Evidently, this technology and its applications need to be discussed in a broad transversal debate that includes scientists, doctors, philosophers, lawyers, patients, politicians, etc. An informed society is empowered to make decisions, especially those that affect the entire future of humanity. Indeed, steps have already been taken in this direction. Serious surveys about what ordinary people think about these matters show that, even in Europe, there are differences in social perception (Gaskell et al., 2017). Although most agree on the use of gene therapy, some countries do not accept genetic enhancement at all. Surprisingly, Spain is much more open to it than other European countries. Several Spanish bioethics institutions have published documents reflecting upon these questions and make several recommendations, for example: not banning genetic editing experimentation on humans, especially in the case of somatic therapy, and not immediately rejecting genetic enhancement in humans (De Lecuona, Casado, Marfany, López-Baroni, \& Escarrabill, 2017). Importantly, a multidisciplinary transEuropean Association for Responsible Research and Innovation in Genetic Editing (ARRIGE) in all organisms, including humans already exists (Montoliu et al., 2018). However, the debate about the bioethical implications of CRISPR-based techniques has only just begun.

\section{HUMANS 2.0}

A famous book by Richard Dawkins is titled The blind watchmaker (in reference to evolution following the mandates of natural selection, not the designs of an intelligent creator), but perhaps 


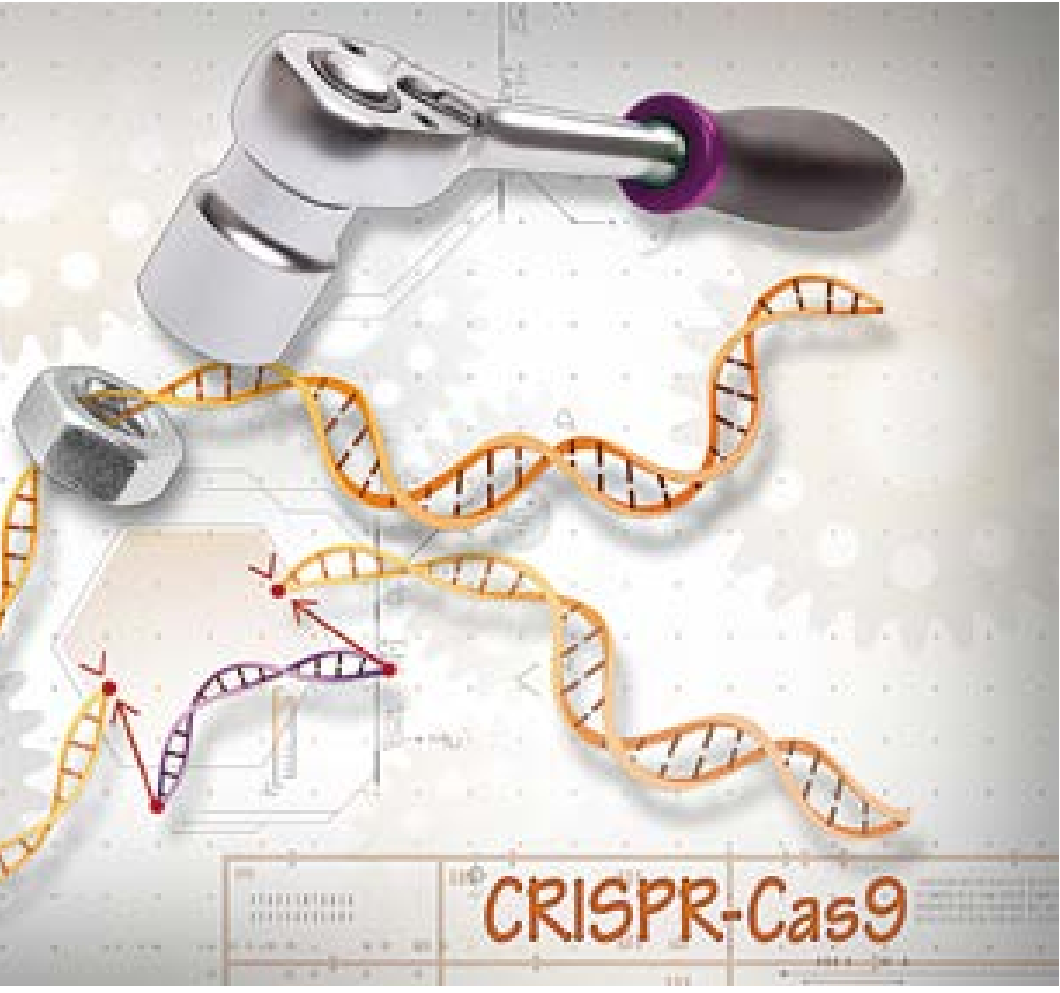

The CRISPR/Cas9 system can correct the genetic mutation that causes a hereditary disease directly in the patient's body.
Gaskell, G., Bard, I., Allansdottir, A., Vieira da Cunha, R., Eduard, P., Hampel, J., ... Zwart, H. (2017). Public views on gene editing and its uses. Nature Biotechnology, 35, 1021-1023. doi: 10.1038/ nbt.3958

Gerbault, P., Liebert, A., Itan, Y., Powell, P., Currat, M., Burger, J. ... Thomas, M. J. (2011). Evolution of lactase persistence: An example of human niche construction. Philosophical Transactions of the Royal Society of London B Biological Sciences, 366(1566), 863877. doi: $10.1098 /$ rstb. 2010.0268

Hirsch, T., Rothoeft, T., Teig, N., Bauer, J. W., Pellegrini, G., De Rosa, L. ... De Luca, M. (2017). Regeneration of the entire human epidermis using transgenic stem cells. Nature, 551, 327-332. doi: 10.1038/nature24487

Kalia, S. S., Adelman, K., Bale, S. J., Chung, W. K., Eng, C., Evans, J. P. ... Miller, D. T. (2017). Recommendations for reporting of secondary findings in clinical exome and genome sequencing, 2016 update (ACMG SF v2.0): A policy statement of the American College of Medical Genetics and Genomics. Genetics in Medicine, 19(2), 249-255. doi: 10.1038/gim.2016.190

Ku, C. S., Polychronakos, C., Tan, E. K., Naidoo, N., Pawitan, Y., Roukos, D. H., ... Cooper D. N. (2013). A new paradigm emerges from the study of de novo mutations in the context of neurodevelopmental disease. Molecular Psychiatry, 18(2), 141-153. doi: $10.1038 / \mathrm{mp} .2012 .58$

Lim, E. T., Uddin, M., De Rubeis, S., Chan, Y., Kamumbu, A. S., Xhang, X., ... Walsh, C. A. (2017). Rates, distribution and implications of postzygotic mosaic mutations in autism spectrum disorder. Nature Neuroscience, 20, 1217-1224. doi: 10.1038/ nn.4598

McCullough, J. M., Heath, K. M., \& Smith, A. M. (2015).

Hemochromatosis: Niche construction and the genetic domino effect in the European Neolithic. Human Biology, 87(1), 39-58. PMID: 26416321

Mojica, F. J. M., \& Montoliu, L. (2016). On the origin of CRISPRCas technology: From prokaryotes to mammals. Trends in Microbiology, 24(10), 811-820. doi: 10.1016/j tim.2016.06.005

we should rewrite it and describe humans as «blind engineers» because we are replacing natural selection to obtain new non-random genetic variants that respond to specific short-term objectives. So far, we have circumvented and modulated natural selection, but genome editing may also become an evolutionary force with specific goals. What are these objectives? We do not yet know, and perhaps we will never know at all. But the future is already here and our genome could begin to change faster than it ever has thanks to the biotechnological tools we have invented. We are now humans 1.0, but perhaps we are at the starting point for humans 2.0. $\odot$

\section{REFERENCES}

Apte, R. S. (2018). Gene therapy for retinal degeneration. Cell, 173(1), 5. doi: 10.1016/j.cell.2018.03.021

Beans, C. (2018). Science and culture: Wearable tech meets tattoo art in a bid to revolutionize both. Proceedings of the National Academy of Sciences of the USA, 115(14), 3504-3506. doi: 10.1073/ pnas.1803214115

De Lecuona, I., Casado, M., Marfany, G., López-Baroni, M., \& Escarrabill, M. (2017). Gene editing in humans: Towards a global and inclusive debate for responsible research. Yale Journal of Biology and Medicine, 90(4), 673-681. PMID: 29259532
Montoliu, L., Merchant, J., Hirsch, F., Abecassis, M., Jouannet, P., Baertschi, B., ... Chneiweiss, H. (2018). ARRIGE arrives: Toward the responsible use of genome editing. The CRISPR Journal, 1(2), 128-129. doi: 10.1089/crispr.2018.29013.mil Roukos, D. H. (2014). Innovation versus evidence: To trust direct-to-consumer personal genomic tests? Expert Review of Molecular Diagnostics, 11, 1-4. doi: 10.1586/erm.10.99

Santander, N. G., Contreras-Duarte, S., Awad, M. F., Lizama, C., Passalacqua, I., Rigotti, A., \& Busso, D. (2013). Developmental abnormalities in mouse embryos lacking the HDL receptor SR-BI. Human Molecular Genetics, 22(6), 1086-1096. doi: $10.1093 / \mathrm{hmg} / \mathrm{dds} 510$

The 1000 Genomes Project Consortium. (2015). A global reference for human genetic variation. Nature, 526, 68-74. doi: 10.1038/ nature15393

Vernot, B., \& Pääbo, S. (2018). The predecessors within... Cell, 173(1), 6-7. doi: 10.1016/j.cell.2018.03.023

Wang, H., La Russa, M., \& Li, Q. S. (2016). CRISPR/Cas9 in genome editing and beyond. Annual Reviews in Biochemistry, 85, 227-264. doi: 10.1146/annurev-biochem-060815-014607

GEMMA MARFANY. Professor of Genetics at the University of Barcelona (Spain), with an extensive scientific and academic career in genetics. She directs a research group investigating the genetic basis of rare hereditary ailments, especially blindness. She is a member of the Institute of Biomedicine (IBUB), member of CIBERER, and of several commissions on bioethics. She is the co-founder of DBGen, a company which performs genetic diagnosis, has written two popular science books, and writes a weekly scientific science column at www.elnacional.cat. \gmarfany@ub.edu 